\title{
Prospective cohort study on mesh shrinkage measured with MRI after laparoscopic ventral hernia repair with an intraperitoneal iron oxide-loaded PVDF mesh
}

\author{
Filip Muysoms $^{1} \cdot$ Roel Beckers $^{2} \cdot$ Iris Kyle-Leinhase ${ }^{1}$
}

Received: 6 August 2017 / Accepted: 19 November 2017 / Published online: 21 December 2017

(c) The Author(s) 2017. This article is an open access publication

\begin{abstract}
Background Current data on shrinkage of intraperitoneal meshes come mainly from animal studies. High-quality human data in prospective studies are scarce.

Methods We used the ability to visualize intraperitoneal PVDF meshes enhanced with iron particles (DynaMesh IPOM visible) with magnetic resonance imaging (MRI) to determine the amount of shrinkage between 1 and 13 months postoperatively. All measurements of the width, length, and surface area of the mesh were performed with a standardized methodology independently by four radiologists blinded for the timing of the MRI.

Results Of the 15 patients undergoing laparoscopic ventral hernia repair, 13 patients received an MRI both at 1 and at 13 months. Evaluation of inter-rater reliability between the radiologists showed intra-class correlations of 0.95 (95\% CI 0.92-0.98) for the width, 0.96 (95\% CI 0.93-0.98) for the length, and 0.99 (90\% CI 0.99-1.00) for the surface area of the mesh. The change between measurement at implantation and 1-month MRI was $-0.7 \mathrm{~cm}(P=0.023 ;-3.6 \%)$ for the width and $-1.9 \mathrm{~cm}(P=0.001 ;-7.2 \%)$ for the length. The change between 1 and 13 months was $-0.06 \mathrm{~cm}(P=0.74$; shrinkage $=0.3 \%)$ for the width, $-0.12 \mathrm{~cm}(P=0.56$; shrinkage $=0.5 \%)$ for the length, and $-4.0 \mathrm{~cm}^{2}(P=0.20 ;$ shrinkage $=1.0 \%)$ for the surface area of the mesh.

Conclusion There is excellent inter-rater reliability between radiologists when measuring width, length, and surface area of visible intraperitoneal PVDF mesh with MRI. There is no significant shrinkage between 1 and 13 months of intraperitoneal PVDF mesh after laparoscopic ventral hernia repair.
\end{abstract}

Keywords Ventral hernia $\cdot$ Laparoscopy $\cdot$ Intraperitoneal mesh $\cdot$ Shrinkage $\cdot$ Magnetic resonance imaging $\cdot$ PVDF

Meeting presentations: The data of the study were presented at the 39th Annual International Congress of the European Hernia Society, May 27, 2017, Vienna, Austria and at the 25th Annual congress of the European Association of Endoscopic Surgery, June 17, 2017, Frankfurt, Germany.

\section{Filip Muysoms}

filip.muysoms@azmmsj.be

1 Department of Surgery, Maria Middelares Hospital, Buitenring Sint-Denijs 30, 9800 Ghent, Belgium

2 Department of Radiology, Maria Middelares, Ghent, Belgium

\section{Background and rationale}

It is believed that meshes used for intraperitoneal laparoscopic ventral hernia repair shrink to a variable extent after implantation [1]. It is therefore considered important to have adequate overlap of the mesh beyond the hernia defect [2]. Data on mesh shrinkage are mainly deduced from animal studies. Frequently, small animals like rats or rabbits have been used and the size of the implanted mesh has been small [3-6]. It is legitimate to ask if and how these results should be interpreted to estimate the shrinkage of mesh after clinical use in humans. Usage of a porcine animal model is limited by the rapid growth of these animals, making it difficult to perform studies with follow-up of longer than a few months [7]. At the University of Aarhus in Denmark, Paul Wara and Hans Friis-Andersen have developed an animal model of laparoscopic ventral hernia repair in sheep, which allows 
implantation of larger mesh sizes and have follow-up for 12-18 months [8, 9]. This model was first used to compare coated polyester mesh (Parietex ${ }^{\mathrm{TM}}$ Composite, Covidien) with composite PVDF-polypropylene mesh (DynaMesh ${ }^{\circledR}-$ IPOM, FEG Textiltechnik) and identified a shrinkage of 41 and $20 \%$, respectively [8]. In a more recent study, several anchoring devices for mesh fixation were compared and it was concluded that the amount of shrinkage depended not only on the mesh properties, but also on the anchoring device used [9].

Limited human data on shrinkage of intraperitoneal meshes are available and most have a high risk of bias due to the retrospective nature. Three retrospective studies evaluated the shrinkage, defined as the decrease in transverse diameter of intraperitoneal ePTFE mesh (DualMesh ${ }^{\mathrm{TM}}$, WL Gore \& Ass), which can be visualized on CT scan and found it to be $7.5,6.7$, and $10.6 \%$, respectively [10-12].

Beldi et al., as part of a randomized clinical trial comparing laparoscopic ventral hernia repair using a coated polypropylene mesh (Parietene ${ }^{\mathrm{TM}}$ Composite, Covidien) either with or without the use of transfacial sutures, measured the mesh surface area with titanium clips applied to the margin of the mesh and visualized with postoperative abdominal $X$-ray [13]. Significantly higher shrinkage of the transverse diameter was documented ( 3.1 vs. $0.1 \% ; P=0.018)$ if no transabdominal sutures were used.

Incorporating iron oxide particles in meshes allows their precise visualization on postoperative magnetic resonance imaging (MRI) examination, thereby allowing in vivo measurement of mesh dimensions at different time points during follow-up [14, 15]. A study in ten patients using MRI to visualize intraperitoneal DynaMesh IPOM at 1 day postoperative and at 3 months showed a decrease in measured mesh surface area compared to the calculated mesh surface area at implantation of 15.6 and $19.1 \%$, respectively [16].

\section{Objectives}

The objective is to evaluate in a clinical setting the shrinkage, defined as the decrease of mesh surface area, between 1 and 13 months after implantation of intraperitoneal iron oxide-impregnated PVDF mesh measured with MRI. Moreover, we want to evaluate the inter-rater reliability among radiologists with regard to measurement with MRI.

\section{Methods}

\section{Study design}

The study is a prospective single-center observational cohort study. The study report was written in accordance with the
STROBE statement (Strengthening the Reporting of Observational Studies in Epidemiology) [17].

\section{Setting}

The study was performed at Maria Middelares Hospital in Ghent, Belgium. All operations were performed by a single surgeon experienced in laparoscopic ventral hernia repair (FM). All postoperative MRIs were coordinated by one radiologist dedicated to abdominal wall imaging (RB). The study was approved by the ethical committee at Maria Middelares Hospital Ghent with the trial number PB/ $\mathrm{nm} / 2013.031$. Patients were invited to a combined clinical assessment and MRI imaging as an outpatient at 1 and 13 months postoperatively. The study protocol was registered at ClinicalTrials.gov (NCT02177214) before the start of the study on June 26, 2014 with the acronym IMAP study.

\section{Participants}

\section{Inclusion criteria}

Individuals who were scheduled for a laparoscopic repair of a midline ventral hernia (European Hernia Society classification, M2-M3-M4 hernias only) [18], agreed to take part in the study, and signed the informed consent comprised the study sample.

\section{Exclusion criteria}

The following constituted our exclusion criteria: patients below 18 years, lateral hernias (L1-L4), subxiphoid hernias (M1), suprapubic hernias (M5), emergency surgery, cleancontaminated or contaminated procedures, ASA score $>4$, pregnancy, life expectancy below 2 years, refusal to sign informed consent, contra-indications for MRI (implanted electrical devices, not MRI-compatible heart valves, large tattoos, large metal implants in the region of interest), and patients with claustrophobia.

\section{Follow-up}

Patients were invited to an outpatient clinical follow-up at 1 and 13 months by the surgeon (FM) and were questioned for abdominal complaints and all adverse events since the last contact. Patients were clinically evaluated in supine and upright position, both at rest and with Valsalva maneuver. They were also invited to complete the Quality of Life questionnaire of the European Registry of Abdominal Wall Hernias (EuraHS QoL score) [19]. An MRI scan was scheduled immediately after the clinical exam. 


\section{Surgical technique}

Patients were operated under general anesthesia with the previously described laparoscopic technique [20]. All patients were treated with an intraperitoneal PVDF meshes enhanced with iron particles (DynaMesh® IPOM visible, FEG Textiltechnik, Aachen, Germany) and fixation was performed with the double crown technique, using absorbable tacks (Securestrap ${ }^{\circledR}$, Ethicon, Johnson \& Johnson, Somerville, NJ, USA). No transabdominal fixation sutures were used.

DynaMesh ${ }^{\circledR}$ IPOM is a composite mesh specifically designed for intraperitoneal hernia repair. It is composed of $88 \%$ polyvinylidene fluoride (PVDF) monofilament on the visceral side and $12 \%$ polypropylene monofilament at the parietal side. The parietal side promotes ingrowth of the mesh to the abdominal wall and the PVDF layer on the visceral side forms a barrier to the intestines. The mesh pore size is in $80 \%>1.0 \mathrm{~mm}$, with an effective porosity of $41 \%$. According to the classification by Amid et al. Dynamesh IPOM classifies as a macroporous mesh [21] and according to the classification of Klinge et al. as a large pore mesh [22].

\section{Imaging technique}

The examination was performed with the patient in prone and feet first position using the table-integrated posterior coil of a 1.5 T magnetic resonance full digital scanner (Philips Ingenia CX). The study protocol takes about 10-12 min and consists of coronal and axial T2, sagittal in-phase FFE, coronal T1 TFE, and coronal 3D T1 non-fatsat. All sequences were performed in free breathing mode increasing patients comfort during the investigation. All images were sent to our institutional PACS system (AGFA Impax 6.5.2.657, AFGA Healthcare NV, Mortsel Belgium).

\section{Variables}

All patient data, operative data, and postoperative data were entered in the prospective EuraHS online database [19]. Meshes were measured before implantation in their widest (horizontal) and longest (vertical) dimension. The "negative" mesh remnants remaining after cutting the mesh to the required size were kept on file for comparison to the MRI measurement if warranted.

\section{Data measurement}

The position of the mesh was evaluated on axial, coronal, and sagittal images. Complications such as seroma, hematoma, or recurrence were excluded using the T2 and T1 images. Morphology and position of adjacent small bowel loops were evaluated to exclude adhesions of small bowel dilatation (Fig. 1). Measurements were performed on sagittal

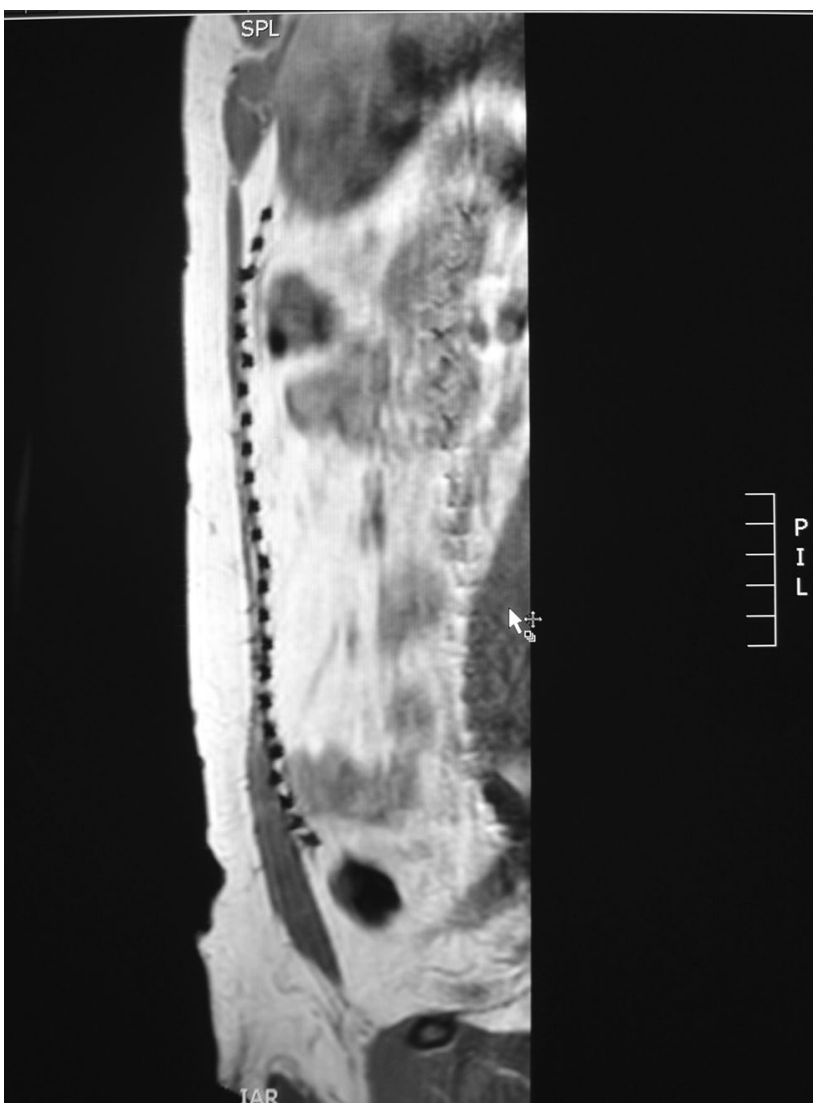

Fig. 1 Native sagittal IF FFE (5 mm slice thickness, TE/TR $4.6 / 329 \mathrm{~ms}, \mathrm{FA} 80^{\circ}$ ) MRI images after laparoscopic intraperitoneal ventral hernia repair with an iron oxide-loaded PVDF mesh

IF FFE series. All patients received sagittal IF FFE on their first and second scan. A 3D T1 low flip angle sequence was included in their second investigation. For blinding, each independent radiologist received a folder in PACS containing the anonymous and randomly ordered scan series.

\section{Quantitative variables}

A 30-mm-thick MINIP (minimal intensity projection) was created in the coronal plane after loading the sagittal IF FFE sequence in an MPR viewer (IMPAX Volume View) followed by drawing the mesh contour along the MR visible wires (Fig. 2). Drawing this contour resulted in projected surface area $\left(\mathrm{mm}^{2}\right)$ and projected circumference $(\mathrm{mm})$. Finally, based on the drawn contour maximal orthogonal diameters $(\mathrm{mm})$ were measured. From the four independent measurements of each scan, the mean value of each variable was calculated as the final outcome.

Quality of Life assessment with the EuraHS QoL instrument was quantified as previously described and presented as median values with interquartile range [23]. 


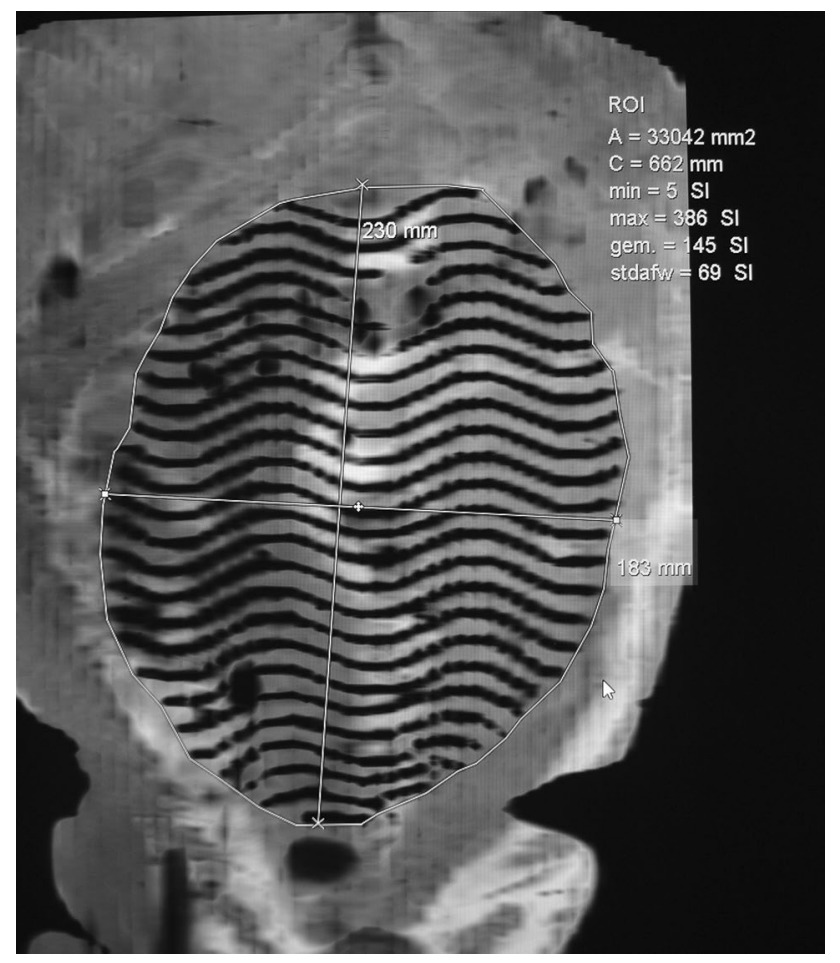

Fig. 2 Mesh surface area was measured on a reconstructed coronal 30-mm-thick MINIP (minimal intensity projection) after loading the sagittal FFE into an MPR viewer (IMPAX Volume View) followed by drawing the mesh contour along the MR visible wires. Drawing this contour resulted in projected surface area $\left(\mathrm{mm}^{2}\right)$ and projected circumference $(\mathrm{mm})$. Based on the drawn contour, maximal orthogonal diameters $(\mathrm{mm})$ were measured

\section{Bias}

To minimize the risk of bias of the measurements taken of the MRI scans, the radiologists were blinded to the patients' identity, the initial measurements of the implanted mesh, and wether the scan was after 1 or 13 months. Moreover, the scans were reviewed in a random sequence avoiding sequential measurements of scans from the same patient.

\section{Study size}

Because no data on mesh shrinkage of implanted intraperitoneal PVDF meshes in humans were available at the start of the study, a sample size of 15 patients was empirically chosen as being large enough to evaluate the mesh shrinkage adequately and small enough to be performed within a reasonable timeframe and within the available budget.

\section{Statistical methods}

The statistical methodology was chosen and performed by an independent statistician. The inter-rater reliability between the four radiologist was estimated by intra-class correlations [ICC $(2,4) ; 95 \% \mathrm{CI}]$ for the width $(\mathrm{cm})$, the length $(\mathrm{cm})$, and the surface area $\left(\mathrm{cm}^{2}\right)$ of the mesh using all 27 available MRI investigations. Inter-rater reliability is considered poor (ICC < 0.40), fair (ICC 0.40-0.59), good (ICC 0.60-0.74), or excellent (ICC 0.75-1.00). Additionally, the ICC was determined separately for the 14 MRI investigations at 1 month and the 13 MRI investigations at 13 months.

The change in the width, length, and surface area of the mesh between 1 and 13 months was estimated as the mean value and standard deviation (SD). A paired $T$ test was used with significance being demonstrated by a $P$ value $<0.05$. Additionally, marginal means and standard error (SE) were estimated using a repeated measures model. Results were reported with a graph using surface area at 1 month $(\mathrm{cm})$ versus change in surface area between 1 and 13 months $(\mathrm{cm})$.

The change in the width and length of the mesh between the measurements at implantation and the MRI measurements at 1 month was estimated as the mean value and SD. A paired $T$ test was again used for analysis with significance being demonstrated by a $P$ value $<0.05$. All statistical analyses were undertaken using SAS statistical software (release 9.4).

\section{Results}

\section{Participants}

A study flow diagram is shown in Fig. 3. During the screening period from June 2014 to August 2015, 18 eligible patients were invited to participate in the study. Two patients declined participation and one patient was excluded intraoperatively as a concomitant parastomal hernia was identified and repaired with a modified Sugarbaker technique. Of 15 patients entered in the study, 13 were evaluated according to protocol with two postoperative MRI scans. One patient became anxious during the procedure and refused further MRI evaluation. One patient declined participation for the second clinical examination and MRI at 13 months. He reported no problems during the telephone contact to plan the follow-up visit.

\section{Descriptive data}

Patient characteristics, intra-operative data, and postoperative clinical outcome are shown in Table 1 . All hernias were midline, either primary $(n=8)$ or incisional $(n=7)$, with a mean width of $3.6 \mathrm{~cm}$ and a mean length of $5.1 \mathrm{~cm}$. The hernia defect was closed in seven patients with a barbed suture, while in smaller hernia defects were not closed. During follow-up, two patients developed an episode of small bowel obstruction needing hospitalization. 
Fig. 3 Study flow diagram of a prospective study on laparoscopic ventral hernia repair with an iron oxide-loaded PVDF mesh to evaluate the mesh shrinkage between 1 and 13 months after implantation

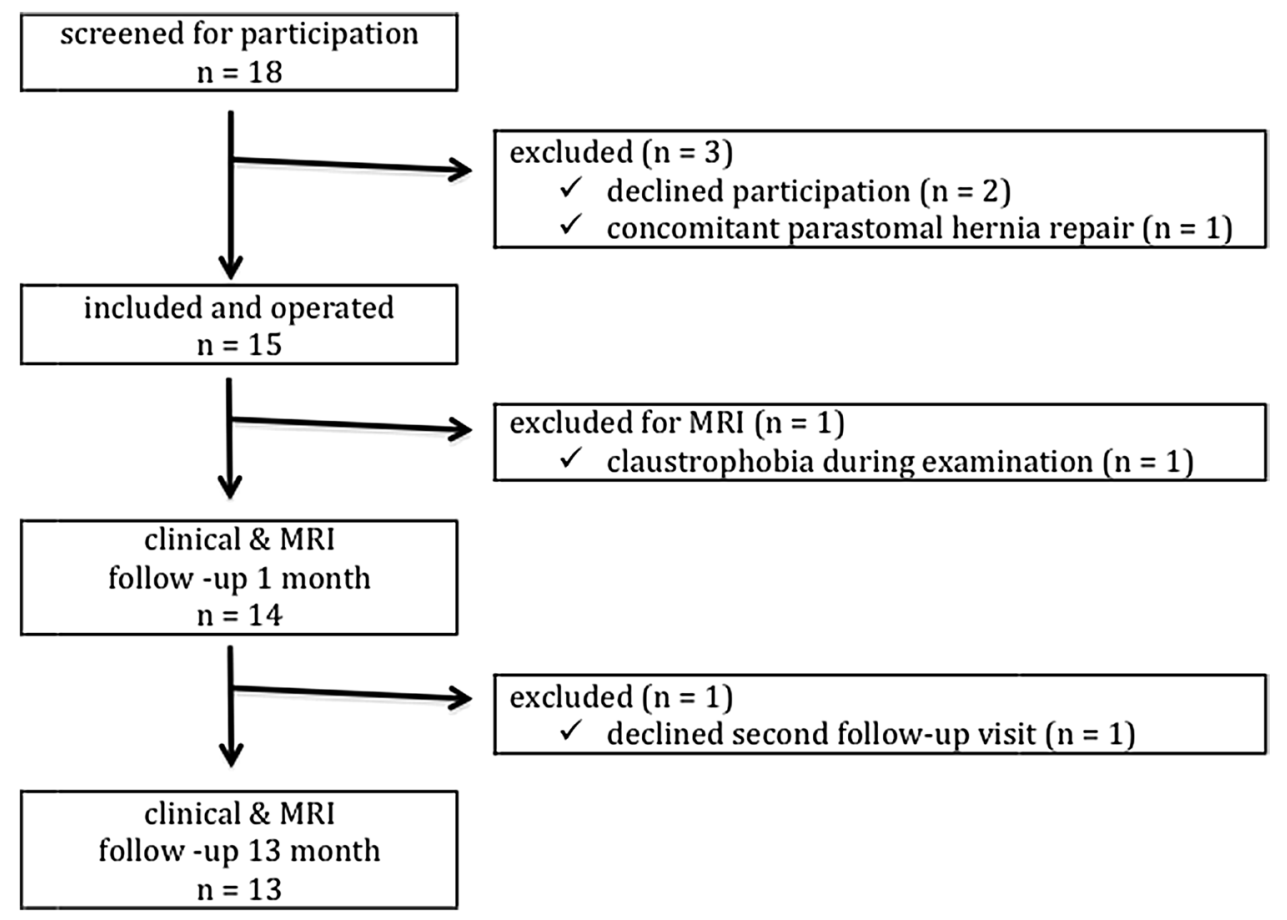

In one patient, this resolved spontaneously and in one patient a laparoscopic release of adhesions to the mesh was needed to resolve the obstruction.

\section{Outcome data}

The outcome data of our study are shown in Table 2. The inter-rater reliability reported as the intra-class correlation was excellent (all mean ICC $\geq 0.94$ ) for the three measurements: width, length, and surface area of the mesh. The changes between measurements at implantation, at 1 , and at 13 months are shown. There is a significant decrease in width $(-0.7 \mathrm{~cm} ; P=0.023)$ and the length $(-1.9 \mathrm{~cm}$; $P=0.001)$ at the 1-month measurement compared to the measurement at implantation. Between 1 and 13 months, no significant change in the width $(-0.06 \mathrm{~cm} ; P=0.740)$, the length $(-0.12 \mathrm{~cm} ; P=0.565)$, or the surface area $\left(-4.0 \mathrm{~cm}^{2} ; P=0.200\right)$ was noted.

\section{Main results}

No significant decrease in mesh surface area (shrinkage) was demonstrated between 1 and 13 months with a mean change in surface area of $-4.0 \mathrm{~cm}^{2}(-1 \%)$. As illustrated in Fig. 4, plotting the change in mesh surface area versus the surface area at 1 month, none of the patients had a shrinkage outside of the boundaries of two SD from the mean.

\section{Other analyses}

The assessment of Quality of Live using the EuraHS QoL instrument showed significant improvement after 1 month compared to the preoperative assessment and a further improvement at 13 months. The results are shown in Fig. 5.

\section{Discussion}

\section{Key result}

There was no significant shrinkage of intraperitoneal PVDF mesh between 1 and 13 months after laparoscopic ventral hernia repair.

\section{Limitations}

The sample size of our study is not very large, but in line with many of the animal studies on mesh shrinkage of intraperitoneal meshes. Nevertheless, since the mean changes in mesh width, length, and surface area were negative, it is likely that a much larger sample size might demonstrate shrinkage to a statistically significant level even with small absolute degrees of shrinkage. On the other hand, the clinical relevance of such a small absolute decrease of mesh surface area is probably low.

We have chosen patients with ventral midline hernias only as this seemed the best population to obtain reproducible and reliable measurements. It is possible that in 
Table 1 Patient characteristics, intra-operative data, and postoperative clinical outcome of a prospective study on laparoscopic ventral hernia repair with an iron oxide-loaded PVDF mesh to evaluate the mesh shrinkage between 1 and 13 months after implantation

\begin{tabular}{|c|c|c|}
\hline & $n / N$ or mean & Range or $\%$ \\
\hline \multicolumn{3}{|l|}{ Patient demographics } \\
\hline Male gender $(n / N)$ & $13 / 15$ & \\
\hline Age (mean/years) & 53 & $31-72$ \\
\hline \multicolumn{3}{|l|}{ Patient variables } \\
\hline Mean BMI $\left(\mathrm{kg} / \mathrm{m}^{2}\right)$ & 29 & $22-37$ \\
\hline Smoker & $5 / 15$ & $33 \%$ \\
\hline Diabetes & $1 / 15$ & $7 \%$ \\
\hline Previous other hernia repair & $3 / 15$ & $20 \%$ \\
\hline \multicolumn{3}{|l|}{ Hernia variables } \\
\hline Primary epigastric & $6 / 15$ & $40 \%$ \\
\hline Primary umbilical & $2 / 15$ & $13 \%$ \\
\hline Incisional hernia & $7 / 15$ & $47 \%$ \\
\hline Width of the hernia $(\mathrm{cm})$ & 3.6 & $1.5-7.2$ \\
\hline Length of the hernia $(\mathrm{cm})$ & 5.1 & $0.7-18.3$ \\
\hline \multicolumn{3}{|l|}{ Operative variables } \\
\hline Duration of surgery $(\mathrm{min})$ & 68 & $55-80$ \\
\hline Defect closure & $7 / 15$ & $47 \%$ \\
\hline Width of the mesh $(\mathrm{cm})$ & 20 & $17-24$ \\
\hline Length of the mesh $(\mathrm{cm})$ & 27 & $21-30$ \\
\hline Operative complications & $3 / 15$ & $20 \%$ \\
\hline Abdominal wall bleeding & 1 & \\
\hline Prolonged ileus & 1 & \\
\hline Constipation & 1 & \\
\hline Hospital stay (days) & 2.5 & $1-7$ \\
\hline \multicolumn{3}{|l|}{ Follow-up at 1 month } \\
\hline $\begin{array}{l}\text { Abdominal wall pain needing medica- } \\
\text { tion }\end{array}$ & $2 / 15$ & \\
\hline $\begin{array}{l}\text { Small bowel obstruction with readmis- } \\
\text { sion }\end{array}$ & $1 / 15$ & \\
\hline Seroma & $0 / 15$ & \\
\hline Surgical site infections & $0 / 15$ & \\
\hline Recurrence & $0 / 15$ & \\
\hline Reoperation & $0 / 15$ & \\
\hline \multicolumn{3}{|l|}{ Follow-up at 13 month } \\
\hline $\begin{array}{l}\text { Small bowel obstruction needing reop- } \\
\text { eration }\end{array}$ & $1 / 15$ & \\
\hline Seroma & $0 / 14$ & \\
\hline Surgical site infections & $0 / 14$ & \\
\hline Recurrence & $0 / 14$ & \\
\hline
\end{tabular}

lateral, subxiphoid, or suprapubic hernias a higher degree of mesh shrinkage would be observed because of the less constant flat positioning of the mesh.

\section{Interpretation}

The degree of shrinkage of PVDF intraperitoneal mesh in our study is much lower than the $20 \%$ shrinkage found in a study on sheep by Zinther et al. [8]. They calculated the shrinkage as the decrease in mesh size between the implanted mesh and the mesh measured up to 18 months postoperatively. Our study notes that there seems to be a discrepancy in mesh size from implantation to 1 month postoperatively. We think this can be attributed to the incomplete flat alignment of the mesh against the abdominal wall during surgery, more than to actual shrinkage after implantation. Moreover, although sheep have a lower growth rate than pigs, the growth was still significant during the study period ( $25 \%$ of weight increase during 12 months), which is not the case for adult humans. They also reported that after the initial shrinkage during the first 3 months no further significant shrinkage was noted.

Comparing our results to the other human study using visible intraperitoneal PVDF mesh, we also found a lower shrinkage rate [16]. Köhler et al. found at 3 months a shrinkage of the mesh surface area of $19 \%$ compared to the implantation size of the mesh. But their baseline MRI on the first postoperative day already demonstrated shrinkage of $15.6 \%$, and thus the majority of the mesh size discrepancy at 3 months should be attributable to the immediate decrease after implantation, probably due to wrinkling of the mesh. The decrease in mesh size in their study between the MRI on postoperative day 1 and at 3 months was only $4.2 \%$.

We think our study shows that after implantation and a period of ingrowth of the mesh during the first month, no additional significant decrease in mesh size is present for intraperitoneal PVDF mesh fixed with a double crown of absorbable tacks. This finding was consistently seen in all the patients, with no outliers demonstrating significant shrinkage. Our study does not support the concept that an individual biological reaction of the patient to the implanted material might cause a highly variable amount of shrinkage between patients for this type of mesh in combination with this type of fixation.

Our findings should not be compared with human studies using intraperitoneal ePTFE mesh due to differences in mesh material, different methodology in mesh size measurement, different definitions for mesh shrinkage, and the high level of bias in most of these studies due to their retrospective nature [10-13].

In our opinion, mesh shrinkage should be determined on measurements of mesh surface area rather than only on the width or the length of the mesh. Mesh shrinkage between two times points $\left(T_{1}\right.$ and $\left.T_{2}\right)$ should be defined as "The percentage of decrease in mesh surface area between two points in time after implantation of the mesh." The method of measurement or calculation of the mesh surface area should 
Table 2 Outcome data of a prospective study on laparoscopic ventral hernia repair with an iron oxide-loaded PVDF mesh to evaluate the mesh shrinkage between 1 and 13 months after implantation with MRI measurement

\begin{tabular}{|c|c|c|c|c|c|}
\hline & & \multicolumn{4}{|c|}{ Intra-class correlation $^{\mathrm{a}}$} \\
\hline & $N$ & Width & \multicolumn{2}{|l|}{ Length } & Surface area \\
\hline All MRI scans & 27 & $0.95(0.92-0.98)$ & \multicolumn{2}{|c|}{$0.96(0.94-0.98)$} & $0.99(0.99-1.00)$ \\
\hline MRI at 1 month & 14 & $0.95(0.89-0.98)$ & \multicolumn{2}{|c|}{$0.99(0.97-1.00)$} & $0.99(0.99-1.00)$ \\
\hline \multirow[t]{4}{*}{ MRI at 13 months } & 13 & $0.96(0.91-0.99)$ & $0.94(0$ & $98)$ & $0.99(0.99-1.00)$ \\
\hline & \multicolumn{5}{|c|}{ Change between measurements at implantation and MRI at 1 month $(N=14$ patients $)$} \\
\hline & Implantation & MRI at 1 month & Difference & Significance & Change $(\%)$ \\
\hline & Mean (SD) & Mean (SD) & Mean (SD) & $P$ value & \\
\hline Width $(\mathrm{cm})$ & $19.9(1.9)$ & $19.1(1.3)$ & $-0.7(0.3)$ & 0.023 & -3.6 \\
\hline \multirow[t]{4}{*}{ Length $(\mathrm{cm})$} & $26.2(2.6)$ & $24.3(2.1)$ & $-1.9(4.5)$ & 0.001 & -7.2 \\
\hline & \multicolumn{4}{|c|}{ Change between MRI at 1 month and MRI at 13 months ( $N=13$ patients) } & \\
\hline & MRI at 1 month & MRI at 13 months & Difference & Significance & Change $(\%)$ \\
\hline & Mean (SD) & Mean (SD) & Mean (SD) & $P$ value & \\
\hline Width $(\mathrm{cm})$ & $19.1(1.4)$ & $19.0(1.3)$ & $-0.06(0.59)$ & 0.740 & -0.3 \\
\hline Length (cm) & $24.4(2.2)$ & $24.2(2.0)$ & $-0.12(0.74)$ & 0.565 & -0.5 \\
\hline Surface area $\left(\mathrm{cm}^{2}\right)$ & $380(44)$ & $376(43)$ & $-4.0(10.5)$ & 0.200 & -1.0 \\
\hline
\end{tabular}

$N$ number of MRI scans available, MRI magnetic resonance imaging, Mean (SD) mean (standard deviation)

${ }^{a}$ Intra-class correlation $=\operatorname{ICC}(2,4)(95 \% \mathrm{CI})$

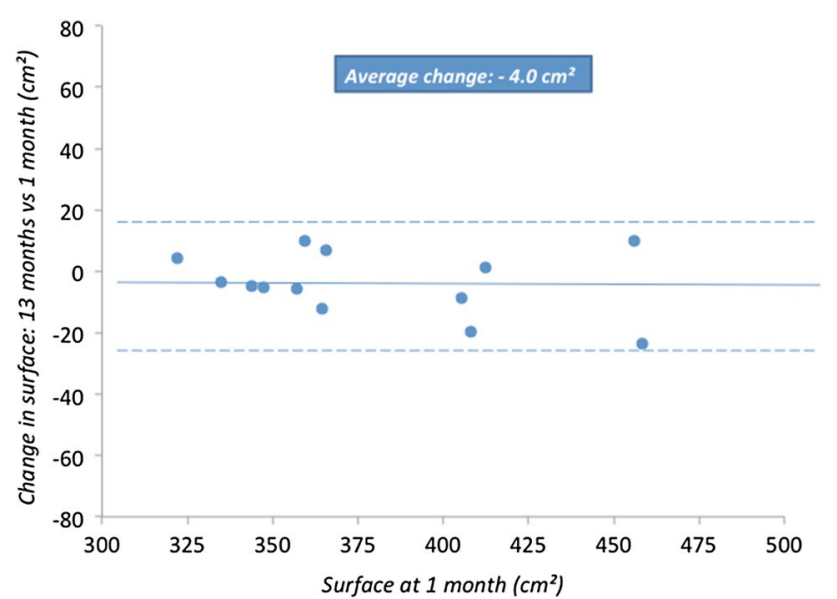

Fig. 4 Graph for the decrease in mesh surface area (shrinkage) as determined with MRI of patients after laparoscopic ventral hernia repair with an iron oxide-loaded PVDF mesh. The change in surface area between 1 and 13 month postoperatively is plotted against the mesh surface area measured at 1 month. The full line indicates the mean decrease in mesh surface area $\left(-0.4 \mathrm{~cm}^{2}\right)$ and the interrupted lines above and below indicate $2 \times \mathrm{SD}$ (standard deviation) from the mean $\left(2 \times 10.5 \mathrm{~cm}^{2}\right)$

be similar for both time points. Comparing a measured mesh surface area after implantation with the calculated mesh surface area at implantation is not considered adequate to detect shrinkage, but it is rather the result of the three-dimensional wrinkling and folding of the mesh during implantation and during release of the pneumoperitoneum.

We propose the following formula for calculating mesh shrinkage, where $T_{1}$ and $T_{2}$ are postoperative selected time points.

Mesh shrinkage between $T_{1}$ and $T_{2}(\%)$

$$
=100-\frac{\text { surface area at } T_{2}\left(\mathrm{~cm}^{2}\right) \times 100}{\text { surface area at } T_{1}\left(\mathrm{~cm}^{2}\right)} \text {. }
$$

Comparing the mesh surface area measured postoperatively to the mesh surface area calculated at implantation and estimating the decrease in effective surface area of the mesh after implantation is interesting to determine the amount of overlap that can be accomplished. But we do not think that this should be labeled as mesh shrinkage.

\section{Generalizability}

Our findings are only applicable to intraperitoneal PVDF mesh fixed with Securestrap absorbable tacks. Other studies have shown that the amount of shrinkage is dependent on the mesh type [8]. Meshes have a large number of variables including the polymer, pore size, weave or knitting details, elasticity, and anti-adhesive coating. These factors may have a significant influence on the rate of shrinkage. 

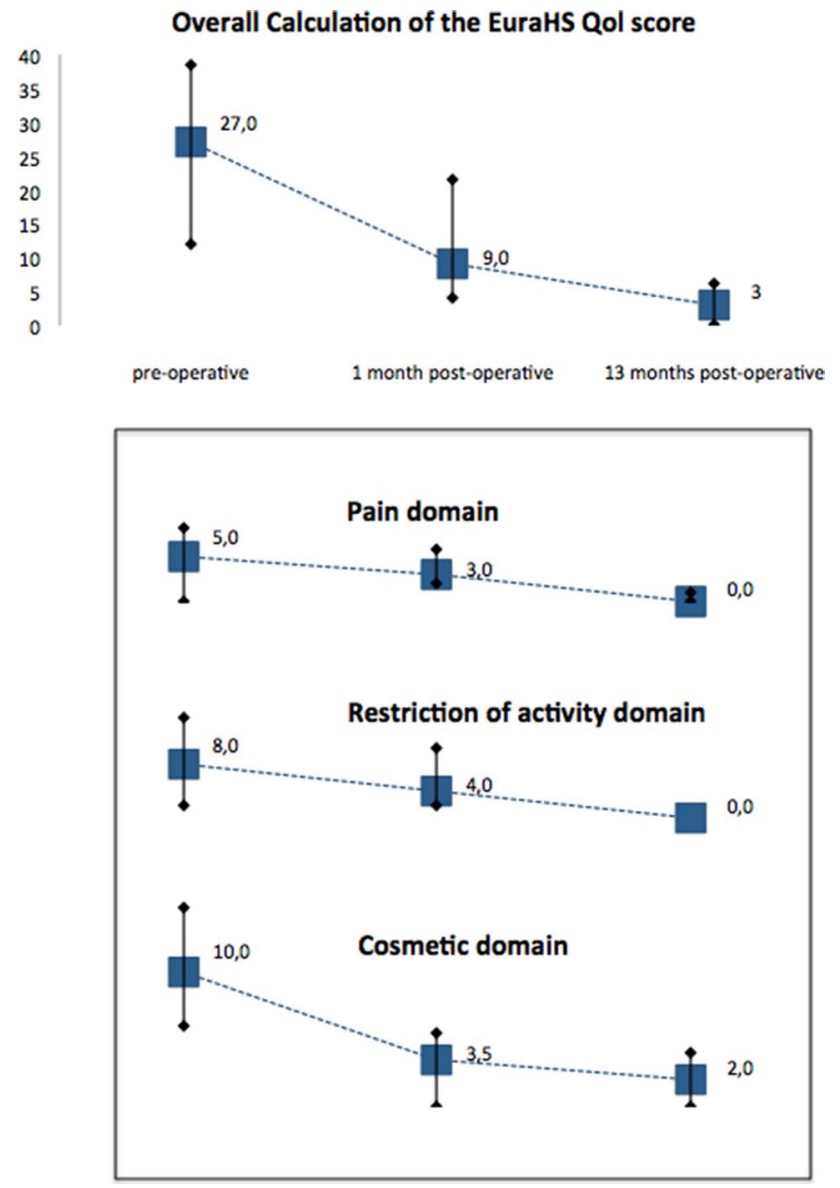

Fig. 5 Graphic report of the Quality of Live assessment with the EuraHS QoL instrument in a prospective study on laparoscopic ventral hernia repair with an iron oxide-loaded PVDF mesh. Results are presented as median values with interquartile ranges

Other studies have shown that the type of fixation also has an important impact on the shrinkage [9, 13]. Beldi et al. found a significantly lower decrease in width of the mesh when transabdominal fixation sutures were added to the tacks fixation [13]. In addition, the mesh position might be important, and our results for intraperitoneal PVDF mesh cannot automatically also be used to estimate the shrinkage rate after retro-muscular mesh placement.

We had two patients with postoperative small bowel obstruction during follow-up, one of them needing a reoperation. The composite PVDF-polypropylene mesh (DynaMesh®-IPOM, FEG Textiltechnik) has demonstrated favorable long-term results after intraperitoneal placement in a prospective observational study by Berger et al. in 344 patients with a mean follow-up of 24 months [24]. Other authors have published some concerns with the intraperitoneal use of this mesh in a much smaller patient cohort of 29 patients [25]. A more recent prospective study by Sommer et al. demonstrated a $6 \%$ reoperation rate after a median follow-up of 36 months, and although some reoperations were because of adhesions, the majority were reoperations for a symptomatic recurrence [26]. In a retrospective study on 88 patients undergoing laparoscopic ventral hernia repair, Tandon et al. compared 62 Parietex $^{\mathrm{TM}}$ Composite meshes and 26 DynaMesh ${ }^{\circledR}$ IPOM meshes [27]. With a median follow-up of 53.6 months, they found a recurrence rate of 12.9 and $3.8 \%$, respectively, for Parietex ${ }^{\mathrm{TM}}$ Composite and DynaMesh® IPOM. DynaMesh® IPOM was associated with a significant higher incidence of intestinal obstructions to adhesions. Our study is too small to add significant knowledge about the frequency of adhesion formation and small bowel obstruction after intraperitoneal PVDF-polypropylene mesh.

\section{Other information}

\section{Study registration}

The study protocol was registered at ClinicalTrials.gov (NCT02177214) before the start of the study on June 26, 2014 with the acronym IMAP study.

Acknowledgements We thank Dr Evert Heindryckx, Dr Kenneth Carels, and Dr Christophe Schoofs from the Department of Radiology at Maria Middelares Hospital Ghent for the independent measurements of the MRI scans.

Funding The study is investigator initiated and was funded with a research grant from Duomed, Londerzele, Belgium for the cost of the MRI investigations and the study insurance. The cost for the independent statistical analysis was funded with a grant from DynaMesh, FEG Textiltechnik, Aachen, Germany. The sponsors were not involved in the initiation, the conduct, or the analysis of the study.

\section{Compliance with ethical standards}

Disclosures Outside the study Filip Muysoms reports research grant and consultancy fees from Medtronic; research grant and speakers fees from Johnson \& Johnson; research grants and speakers fees from DynaMesh; proctoring contract and consultancy for Intuitive Surgical. Iris Kyle-Leinhase and Roel Beckers have no conflicts of interest or financial ties to disclose.

Open Access This article is distributed under the terms of the Creative Commons Attribution 4.0 International License (http://creativecommons.org/licenses/by/4.0/), which permits unrestricted use, distribution, and reproduction in any medium, provided you give appropriate credit to the original author(s) and the source, provide a link to the Creative Commons license, and indicate if changes were made. 


\section{References}

1. Jonas $\mathbf{J}$ (2009) The problem of mesh shrinkage in laparoscopic incisional hernia repair [Article in German]. Zentralbl Chir 134:209-213

2. LeBlanc K (2016) Proper mesh overlap is a key determinant in hernia recurrence following laparoscopic ventral and incisional hernia repair. Hernia 20:85-99

3. Harrell AG, Novitsky YW, Peindl RD, Cobb WS, Austin CE, Cristiano JA, Norton JH, Kercher KW, Heniford BT (2006) Prospective evaluation of adhesion formation and shrinkage of intraabdominal prosthetics in a rabbit model. Am Surg 72:808-813

4. Judge TW, Parker DM, Dinsmore RC (2007) Abdominal wall hernia repair: a comparison of sepramesh and parietex composite mesh in a rabbit hernia model. J Am Coll Surg 204:276-281

5. Celik A, Altinli E, Koksal N, Celik AS, Onur E, Ozkan OF, Gumrukcu $G$ (2009) The shrinking rates of different meshes placed intraperitoneally: a long-term comparison of the TiMesh, VYPRO II, Sepramesh, and DynaMesh. Surg Laparosc Endosc Percutan Tech 19:e130-e134

6. Chatzimavroudis G, Kalaitzis S, Voloudakis N, Atmatzidis S, Kapoulas S, Koutelidakis I, Papaziogas B, Christoforidis EC (2017) Evaluation of four mesh fixation methods in an experimental model of ventral hernia repair. J Surg Res 212:253-259

7. Schug-Pass C, Sommerer F, Tannapfel A, Lippert H, Köckerling F (2009) The use of composite meshes in laparoscopic repair of abdominal wall hernias: are there differences in biocompatibily?: experimental results obtained in a laparoscopic porcine model. Surg Endosc 23:487-495

8. Zinther NB, Wara P, Friis-Andersen H (2010) Shrinkage of intraperitoneal onlay mesh in sheep: coated polyester mesh versus covered polypropylene mesh. Hernia 14:611-615

9. Harsløf S, Zinther N, Harsløf T, Danielsen C, Wara P, FriisAndersen H (2017) Mesh shrinkage depends on mesh properties and anchoring device: an experimental long-term study in sheep. Hernia 21:107-113

10. Schoenmaeckers EJ, van der Valk SB, van den Hout HW, Raymakers JF, Rakic S (2009) Computed tomographic measurements of mesh shrinkage after laparoscopic ventral incisional hernia repair with an expanded polytetrafluoroethylene mesh. Surg Endosc 23:1620-1623

11. Carter PR, LeBlanc KA, Hausmann MG, Whitaker JM, Rhynes VK, Kleinpeter KP, Allain BW (2012) Does expanded polytetrafluoroethylene mesh really shrink after laparoscopic ventral hernia repair? Hernia 16:321-325

12. Tsuruta A, Hirai T, Nakamura M (2014) Retrospective comparison of open versus laparoscopic ventral and incisional hernia repair. Asian J Endosc Surg 7:246-250

13. Beldi G, Wagner M, Bruegger LE, Kurmann A, Candinas D (2011) Mesh shrinkage and pain in laparoscopic ventral hernia repair: a randomized clinical trial comparing suture versus tack mesh fixation. Surg Endosc 25:749-755

14. Kuehnert N, Kraemer NA, Otto J, Donker HC, Slabu I, Baumann M, Kuhl CK, Klinge U (2012) In vivo MRI visualization of mesh shrinkage using surgical implants loaded with superparamagnetic iron oxides. Surg Endosc 26:1468-1475

15. Ciritsis A, Hansen NL, Barabasch A, Kuehnert N, Otto J, Conze J, Klinge U, Kuhl CK, Kraemer NA (2014) Time-dependent changes of magnetic resonance imaging-visible mesh implants in patients. Invest Radiol 49:439-444

16. Köhler G, Pallwein-Prettner L, Koch OO, Luketina RR, Lechner M, Emmanuel K (2015) Magnetic resonance-visible meshes for laparoscopic ventral hernia repair. JSLS 19:e2014.00175

17. von Elm E, Altman DG, Egger M, Pocock SJ, Gøtzsche PC, Vandenbroucke JP (2007) Strengthening the reporting of observational studies in epidemiology (STROBE) statement: guidelines for reporting observational studies. BMJ 335:806-808

18. Muysoms F, Miserez M, Berrevoet F, Campanelli G, Champault GG, Chelala E, Dietz UA, Eker HH, El Nakadi I, Hauters P, Hidalgo Pascual M, Hoeferlin A, Klinge U, Montgomery A, Simmermacher RK, Simons MP, Smietański M, Sommeling C, Tollens T, Vierendeels T, Kingsnorth A (2009) Classification of primary and incisional abdominal wall hernias. Hernia 13:407-414

19. Muysoms F, Campanelli G, Champault GG, DeBeaux AC, Dietz UA, Jeekel J, Klinge U, Köckerling F, Mandala V, Montgomery A, Morales Conde S, Puppe F, Simmermacher RK, Śmietański M, Miserez M (2012) EuraHS: the development of an international online platform for registration and outcome measurement of ventral abdominal wall hernia repair. Hernia 16:239-250

20. Muysoms FE, Kyle-Leinhase I, Novik B, Berrevoet F (2012) Mesh fixation alternatives for laparoscopic ventral hernia repair. Surg Technol Int 22:125-132

21. Amid P (1997) Classification of biomaterials and their related complications in abdominal wall surgery. Hernia 1:15-21

22. Klinge U, Klosterhalfen B (2012) Modified classification of surgical meshes for hernia repair based on the analyses of 1000 explanted meshes. Hernia 16:251-258

23. Muysoms FE, Vanlander A, Ceulemans R, Kyle-Leinhase I, Michiels M, Jacobs I, Pletinckx P, Berrevoet F (2016) A prospective, multicenter, observational study on Quality of Life after laparoscopic inguinal hernia repair with ProGrip ${ }^{\mathrm{TM}}$ laparoscopic self-fixating mesh according to the EuraHS-QoL instrument. Surgery 160:1344-1357

24. Berger D, Bientzle M (2009) Polyvinylidene fluoride: a suitable mesh material for laparoscopic incisional and parastomal hernia repair! A prospective, observational study with 344 patients. Hernia 13:167-172

25. Fortelny RH, Petter-Puchner AH, Glaser KS, Offner F, Benesch T, Rohr M (2010) Adverse effects of polyvinylidene fluoride-coated polypropylene mesh used for laparoscopic intraperitoneal onlay repair of incisional hernia. Br J Surg 97:1140-1145

26. Sommer T, Friis-Andersen H (2013) DynaMesh® in the repair of laparoscopic ventral hernia: a prospective trial. Hernia 17:613-618

27. Tandon A, Shahzad K, Pathak S, Oommen CM, Nunes QM, Smart N (2016) Parietex ${ }^{\mathrm{TM}}$ composite mesh versus Dynamesh $®-I P O M$ for laparoscopic incisional and ventral hernia repair: a retrospective cohort study. Ann R Coll Surg Engl 98:568-573 